\title{
Diffusion with social reinforcement: The role of individual preferences
}

\author{
Elena M. Tur* \\ INGENIO (CSIC-UPV), Universitat Politècnica de València, Camino de Vera s/n, E-46022 Valencia, Spain; \\ School of Innovation Sciences, Eindhoven University of Technology, Eindhoven, The Netherlands; \\ and Institute of Innovation and Entrepreneurship, School of Business, Law and Economics, University of Gothenburg, Gothenburg, Sweden \\ Paolo Zeppini \\ Department of Economics, University of Bath, Bath, England, United Kingdom \\ Koen Frenken \\ Innovation Studies, Copernicus Institute of Sustainable Development, Utrecht University, Utrecht, The Netherlands
}

(Received 20 March 2017; published 6 February 2018)

\begin{abstract}
The debate on diffusion in social networks has traditionally focused on the structure of the network to understand the efficiency of a network in terms of diffusion. Recently, the role of social reinforcement has been added to the debate, as it has been proposed that simple contagions diffuse better in random networks and complex contagions diffuse better in regular networks. In this paper, we show that individual preferences cannot be overlooked: complex contagions diffuse better in regular networks only if the large majority of the population is biased against adoption.
\end{abstract}

DOI: 10.1103/PhysRevE.97.022302

\section{INTRODUCTION}

Diseases, ideas, and behaviors often spread through social contact [1]. A main concern in these cases is how far the diffusion process will reach, either to promote behaviors of a healthy lifestyle, to foster innovation adoption, or to prevent criminal behavior or an epidemic outbreak. In all these cases, the question is about how many agents will "adopt," and how the social network structure determines the diffusion size. In the context of information diffusion, Granovetter [2] introduced the novel idea that "weak ties" (shortcuts between otherwise unconnected people) can foster diffusion by connecting distant people, and allowing individuals to access novel sources, so that information can travel a longer social distance and reach a broader audience. Conversely, "strong ties" (transitive ties between people with common acquaintances) are redundant, since the information that a very close friend can bring is not very different from that accessible to any number of our common friends.

For many years, weak ties have been considered as the main driver of diffusion. Indeed, they have been proven important in processes like job finding [2] and disease spreading [3]. Some studies even found that the weight distribution was more important than the topological structure of the network in determining the diffusion pattern [4]. Weak ties have also been proven crucial in link prediction problems [5].

More recently, Centola et al. [6] introduced an additional dimension in the study of diffusion processes: social reinforce-

\footnotetext{
*e.m.mas.tur@tue.nl
}

Published by the American Physical Society under the terms of the Creative Commons Attribution 4.0 International license. Further distribution of this work must maintain attribution to the author(s) and the published article's title, journal citation, and DOI. ment. They call "complex propagation" those cases of diffusion where social reinforcement increases locally the probability of transmission, as opposed to "simple propagation," where social reinforcement is irrelevant. In the latter case, weak ties represent a crucial component of diffusion, because the first contact is enough to trigger contagion. In cases of complex propagation, by contrast, additional contacts increase the probability of contagion through social reinforcement. Thus strong ties are not redundant, since they can prompt a cascade dynamic. Social reinforcement changes the effect of the network structure of social contacts where diffusion takes place: while diffusion processes of simple propagation reach a higher number of agents in random networks with many shortcuts, complex propagation processes work better in ordered and clustered networks such as ring lattices.

Following the seminal work by Granovetter [7], Centola et al. [6] introduced a threshold model of diffusion in a social network. An agent's decision rule is to adopt if (and only if) the portion of adopting neighbors relative to the size of the neighborhood is over a certain threshold $T$. This threshold remains constant for all agents. They compare different social structures such as ring lattices, small worlds, random networks, and scale-free networks. Their main conclusion is that complex propagations (those where multiple contacts increase the likelihood of adoption) are influenced by the existence of multiple short paths between agents more than by single dyadic links. Random bridges can stimulate both simple and complex propagations in small worlds, but they may also inhibit complex propagations for thresholds close to a critical value.

Later that year, Centola and Macy [8] developed their theory, with the same threshold model, from a sociological point of view. Their main message is that, while weak ties promote simple diffusions, strong ties stimulate diffusion of complex propagations. In simple diffusions, only the first contact with the disseminule is relevant: transitive ties between friends 
are thus redundant. By contrast, in complex propagations diffusion benefits from reinforcement from multiple sources: this transitive structure is now a pathway for diffusion.

In 2010, Centola [9] explored this result in an online experimental setting. Participants were connected anonymously through different social structures and faced a decision to adopt a behavior, namely, to register in a health forum. Participants received a message informing them that other participants in their ego network (their "health buddies") had adopted the behavior, every time a health buddy adopted. Again, locally clustered ties seemed to be highly efficient for stimulating the diffusion of a behavior.

The experiments received a lot of attention, since it pointed to a key issue on the debate of which network structure is more efficient in terms of diffusion: that it depends on what is being diffused. It criticized that diffusion through social contact had been generalized to "whatever was being diffused" (their emphasis, paraphrasing Granovetter [2]). The result showed that depending on the type of information that was being diffused (either simple, or complex needing confirmation from multiple sources), the role of weak ties and strong ties changed. While for simple contagions strong ties were redundant, for complex propagations they allowed for reinforcing confirmation from additional peers. Once a node in the neighborhood adopts, the likelihood is high that this node will share several common contacts with the focal agent that will reinforce the message (if they adopt as well).

In 2013, Zheng et al. [10] proposed a model of diffusion that could replicate the results of the experiment by Centola [9]. In order to introduce social reinforcement, they defined the probability of adoption as dependent on the number of contacts with the disseminule, according to a certain social reinforcement intensity. This probability of adoption after a certain number of contacts is kept constant for all agents in the network. They calibrate the parameters of the model to replicate the adoption proportion found by Centola [9], and conclude that, indeed, under social reinforcement regular (clustered) networks promote diffusion better than random networks. The effect of social reinforcement has also been tested in diffusion processes without an explicit network [11].

Our model below, instead, uses a percolation model, which has been be applied to information diffusion through word of mouth [12-14]. Percolation theory was introduced in physics to study the process of liquids percolating through layers of porous materials. In our setting, agents are connected through a social network. Once an agent gets informed, she then decides whether to adopt according to a decision rule. Only if she decides to adopt, she informs her neighbors who have not yet adopted. In turn, these agents become informed and have to decide whether to adopt.

This process is exactly the same as the diffusion process modeled in the aforementioned models of Centola et al. [6], Centola and Macy [8], and Zheng et al. [10] and the same process through which diffusion took place in the experiments of Centola [9]. The difference, however, between these models and ours lies in the decision rule of agents. Both Centola et al. [6] and Centola and Macy [8] used a threshold model: agents decide to adopt if more than a fraction $T$ of their neighbors had adopted. For Zheng et al. [10], adoption is probabilistic: after $m$ events of local information agents adopt with a certain probability $\lambda_{m}$. In all these cases, agents decide solely on the basis of the number of neighbors who have already adopted.

In this paper, we argue that one dimension of social diffusion still remains underspecified: individual preferences of agents. In our model, we introduce heterogeneous preferences in the context of the adoption of new ideas, although our model equally applies to other diffusion processes. Some agents can be relatively "open," more susceptible and more likely to adopt a new idea at the first contact, while others can be more "closed," less susceptible, and need additional contacts to adopt a new idea. A percolation model of social diffusion in networks implies already a basic form of heterogeneity, since agents' adoption thresholds are drawn from a uniform distribution [1214]. Here we study extensively the effects of heterogeneous preferences by considering nonuniform distributions, and we introduce social reinforcement by lowering this threshold with every additional contact.

With a uniform distribution of preferences, we find that both simple and complex propagations diffuse to a higher number of adopters in random networks than in clustered networks. While cases of complex propagation do benefit from the redundancy that strong ties bring, this effect is not strong enough to counterbalance the influence of weak ties. But when we consider nonuniform distributions of preferences, this effect is altered. With open-minded populations (those with a larger probability mass on low values of the threshold for adoption) random networks still perform better than regular networks, at any level of social influence. However, with closed-minded populations (where most of the agents have a relatively high threshold) regular networks are more efficient in the diffusion process when social influence is strong enough, which is the result of Centola et al. [6].

The main message of the paper, then, is that the distribution of preferences in a process of social diffusion strongly matters, together with the social network structure, when the adoption mechanism occurs by word of mouth and is affected by social influence.

The rest of the paper is structured as follows. Section II describes our model of diffusion on networks with social reinforcement and heterogeneous preferences. Section III presents simulations of the model. Section IV concludes.

\section{OUR MODEL}

\section{A. Percolation with social reinforcement}

Processes of social diffusion share many characteristics with epidemic outbreaks of infectious diseases. Consider a new idea being proposed by one or more individuals in a population. Assume that a quality dimension can be defined for the idea, for example, its originality, plausibility, or usefulness. To the extent that a one-dimensional space captures the quality of the idea, this can be defined as the scalar $\xi \in[0,1]$. A quality equal to zero means a completely unattractive idea, which implies that no one wants to adopt. An idea of quality 1, on the contrary, is an idea that everybody in the population is willing to adopt.

A percolation setting allows for a straightforward introduction of heterogeneity of preferences across the population. Assume that agents are characterized by an individual threshold value for quality, a minimum quality requirement (MQR). This individual threshold is the minimal description 

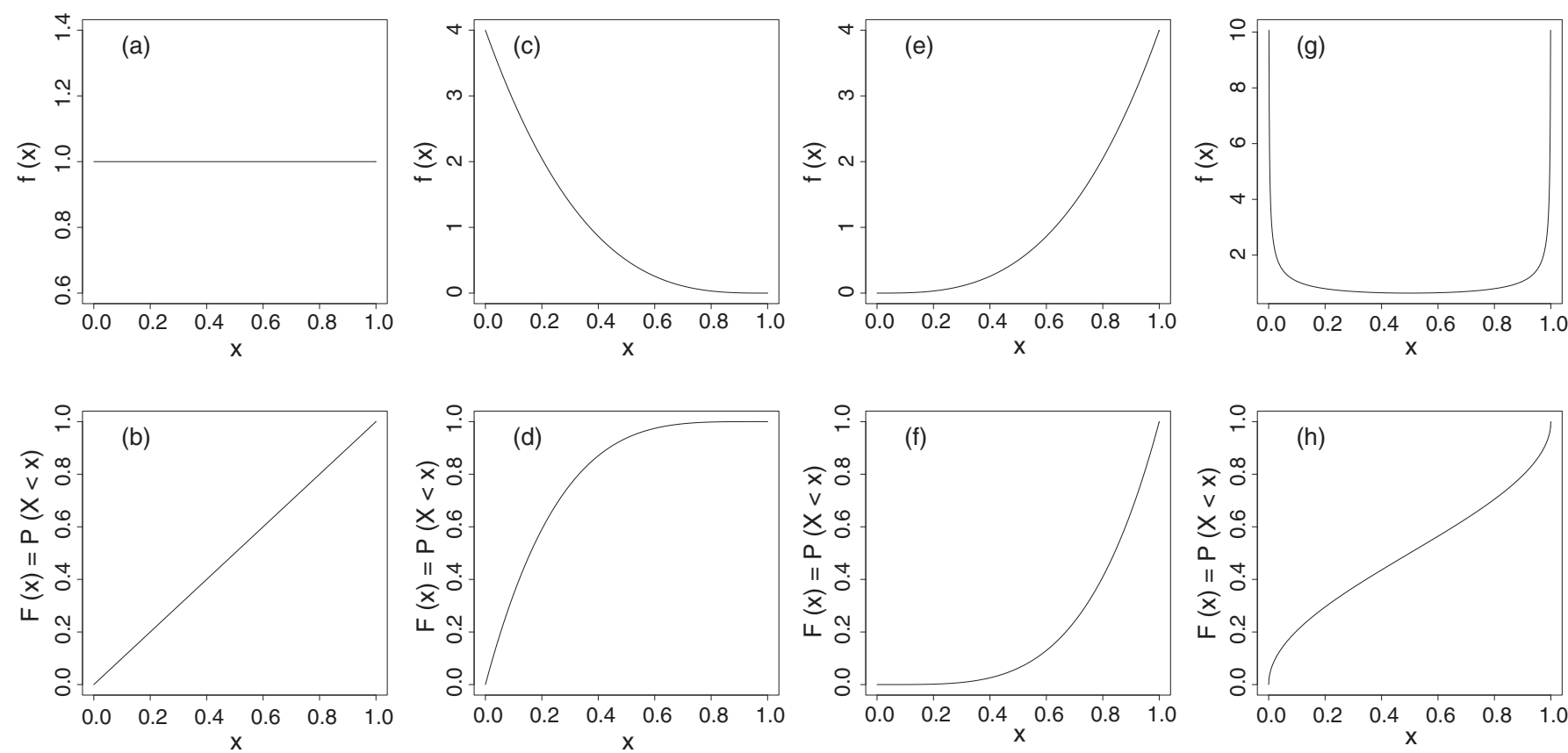

FIG. 1. Different MQR distributions from the $B(\alpha, \beta)$ family: (a, b) $B(1,1)=U(0,1),(\mathrm{c}, \mathrm{d}) B(1,4),(\mathrm{e}, \mathrm{f}) B(4,1)$, and $(\mathrm{g}, \mathrm{h}) B(0.5,0.5)$.

of individual preferences. Note that if we do not talk about the minimum quality of an idea but, reversely, about the maximum price an agent is willing to pay for an innovation, this setting would be analogous to the concept of reservation price in economics [15].

After an idea with a given intrinsic quality is proposed by someone in the population, agents who get to know about the idea by social contact decide whether to adopt or not depending on whether its quality is above or below their MQR. Agents with higher MQR will be less likely to adopt new ideas. Drawing MQR from different distributions allows us to compare populations with different traits, and to study the role of preferences in the process of diffusion in social networks.

We use the notation $\xi \in[0,1]$ for the intrinsic quality of an idea and $q_{t}^{i} \in[0,1]$ for the MQR of agent $i$ at time $t$. In the basic percolation model this individual threshold remains constant over time, that is to say, $q_{t}^{i}=q_{0}^{i} \forall t$. In the basic model, then, the number of adopting neighbors does not play any role in adoption decisions. Nothing changes for an agent if she knows about the new idea from just one or multiple neighbors: the number of adopting neighbors does not carry any influence, and additional adoptions only convey redundant information. In an extension to the percolation model we include a new factor in the expression of the individual valuation of an idea, according to which decisions are influenced by the number of adopting neighbors. Put differently, adopting neighbors can "advocate" in favor of the new idea, so as to increase the likelihood of its adoption.

This updated MQR needs to satisfy the following requirements: (a) it must be decreasing in the number of adopting neighbors, and (b) it must be decreasing in the intensity of social reinforcement. Let $q_{t}^{i} \in[0,1]$ be the MQR of an agent, $a_{t}^{i} \in \mathbb{N}$ the number of adopting neighbors, and $\gamma \geqslant 0 \mathrm{a}$ parameter expressing the social reinforcement intensity. For the new results to be comparable to the basic percolation benchmark, we add the following requirements: (c) for $\gamma=0$ (no social reinforcement), $q_{t}^{i}=q_{0}^{i} \forall t$, and (d) when only one neighbor adopts, $q_{t}^{i}=q_{0}^{i}$. Condition (c) guarantees that the basic percolation model is a particular case of the extended model with social reinforcement, while condition (d) ensures that the first decision to adopt (i.e., after the initial contact with the idea) is the same in both models.

We introduce social reinforcement in the percolation model with the following definition of MQR for each adoption:

$$
q_{t}^{i}=q_{0}^{i}\left(\frac{1}{a_{t}^{i}}\right)^{\gamma} .
$$

Equation (1) describes how the MQR $q_{t}^{i}$ of an agent decreases with the number of adopters $a_{t}^{i}$ in the neighborhood. The function $f(q ; a, \gamma)=q \cdot\left(\frac{1}{a}\right)^{\gamma}$ satisfies the following properties.

(a) $f$ is decreasing in $a$, for $\gamma>0$.

(b) $f$ is decreasing in $\gamma$, for $a>1$.

(c) $f(q ; a, 0)=q$.

(d) $f(q ; 1, \gamma)=q$.

Moreover, we restrict ourselves to $\gamma \leqslant 1$, assuming that the marginal effect of each additional neighbor on the total social influence cannot be increasing. Note here that an infinite value of $\gamma$ would imply that all agents would adopt after at most two neighbors have adopted, which implies that an idea would always diffuse completely in a ring lattice with degree 4 or higher. Since the first adopting neighbor informs each agent, we assume that $f$ is only evaluated after the first neighbor adopts, i.e., $a \geqslant 1$. This means that $f:[0,1] \longrightarrow[0,1]$.

In the model of percolation with social reinforcement described by Eq. (1) the neighbors of an agent's social network always give positive information about the new idea: the more neighbors adopt, the more prone an agent is to adopt. Social reinforcement is a positive force for adoption. The parameter $\gamma$ represents the "intensity" of social influence: for a given number of adopting neighbors, the updated value of MQR 

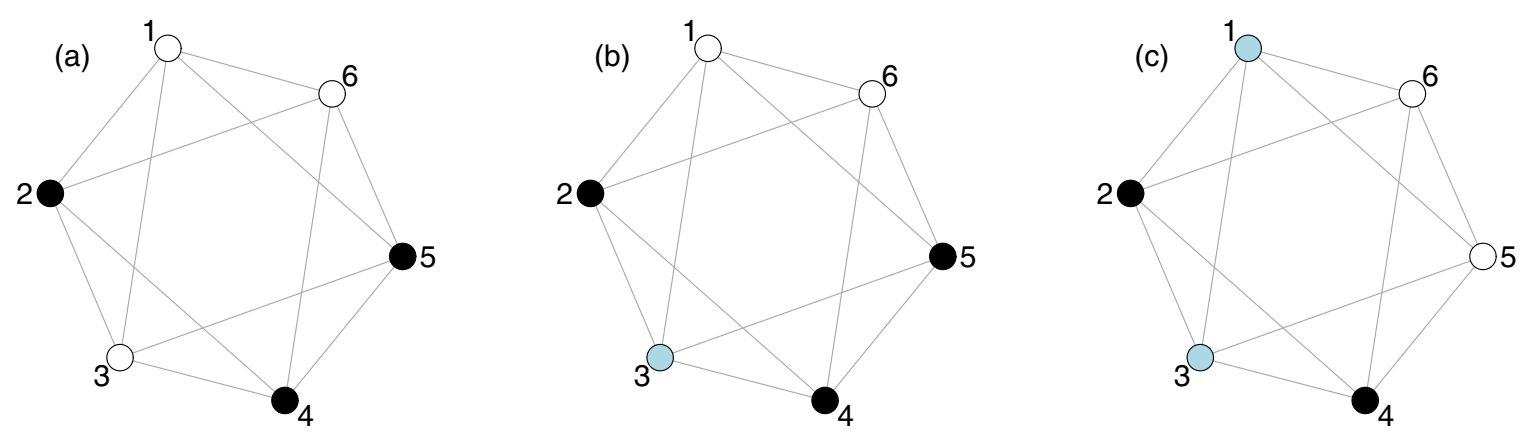

FIG. 2. The effect of social reinforcement on clustered neighborhoods. (a) The white nodes represent the willing to adopt agents. Black nodes represent the unwilling to adopt agents. (b) A willing to adopt agent adopts (blue node, gray in print). (c) The clustering links make social reinforcement more intense for nonwilling to adopt neighbors: node 1 adopts and node 5 becomes willing to adopt.

will be lower for higher $\gamma$, so adoption will occur more often. An agent makes her first adoption decision only after the first adoption event in her neighborhood. The crucial point of social reinforcement is that, contrary to the basic percolation model, this decision is not definitive. An agent may not adopt the innovative idea the first time she is informed, but she may nevertheless adopt later, if further adoption events occur in her neighborhood.

\section{B. Preferences}

Studies on complex propagation so far considered that agents are homogeneous in their willingness to adopt $[6,16]$. We instead follow the basic percolation model, which assumes heterogeneous but uniformly distributed preferences of agents [15].

The marginal effect of an additional adopting neighbor on the MQR of an agent can be evaluated by differentiating Eq. (1) with respect to the number of adopting neighbors, as if they were a continuous variable:

$$
\Delta q_{t}^{i}=q_{0}^{i} \frac{\partial}{\partial a_{t}^{i}}\left(a_{t}^{i}\right)^{-\gamma} \Delta a_{t}^{i}=-\frac{q_{0}^{i} \gamma}{\left(a_{t}^{i}\right)^{\gamma+1}} \Delta a_{t}^{i} .
$$

The first adopting friends induce a large decrease in the $M Q R$, while, after a large number of friends have adopted, the influence of an additional adopting neighbor is negligible. However, the marginal effect $\Delta q_{t}^{i}$ also depends on the individual agent's intrinsic value of the MQR, and it is larger for large values of $q_{0}^{i}$. This is why the distribution of $q_{0}^{i}$ values affects the social influence factor of complex diffusion processes.

The basic percolation model is a limited model in that it only considers a uniform distribution of preferences among agents. Below, we also study percolation with social reinforcement with nonuniform distributions of agents' intrinsic MQR.

The $B(\alpha, \beta)$ family of distributions is particularly suited to our model, since it has support in the interval $[0,1]$. The parameters $\alpha$ and $\beta$ allow us to change the mean and standard deviation of the distribution, and also to vary its symmetry. As before, the cumulative distribution indicates the potential adoption base in a fully mixed and fully informed population. Figure 1 shows some examples of $B(\alpha, \beta)$ distributions. The uniform distribution is a particular case of the Beta family, with $\alpha=\beta=1$ [Figs. 1(a) and 1(b)]. The case of $B(1,4)$ describes a population where most agents have a low value of MQR [Figs. 1(c) and 1(d)]. In contrast, the case of $B(4,1)$ models the opposite situation, where most agents have a large MQR [Figs. 1(e) and 1(f)]. In the context of our model, the latter case is the one of a population where most people are closed minded, or resistant to the adoption of a new idea, and only few people are enthusiastic early adopters. We may consider this one as a realistic case [17-19], where most of a population is reluctant to changes and to embrace novelties, due to reasons that span from psychology (status-quo bias) or sociology (norms) to any fixed costs of investments into physical and human capital, like technological standards and infrastructures. The last case is a polarized population, with $B(0.5,0.5)$, with most individuals either very open minded or very closed minded [Figs. $1(\mathrm{~g})$ and $1(\mathrm{~h})]$. This is a mixture between the two previous extreme populations.

\section{Networks}

To compare diffusion processes on different network topologies, we follow the small world model of Watts and Strogatz [20], which provides one with a family of networks ranging from regular lattices to random networks. The algorithm starts with a regular ring lattice and rewires every link with probability $\mu$. This parameter allows us to fine tune the randomness/clustering of the network. In particular, the rewiring probability determines the number of triads (sets of triangular structures) in the network, also called clusters. The clustering coefficient of a network is the relative number of triads present in the network. In clustered networks, the probability of two agents being connected increases if they have a shared neighbor.

By changing the rewiring probability $\mu$ of the small world algorithm we obtain networks with different average path length and clustering coefficient. The case with $\mu=0$ is the one-dimensional regular lattice, and the case with $\mu=1$ is the random network, also known as the Poisson network or Erdos-Renyi model [21]. For intermediate values of the probability $\mu$, the resulting networks present intermediate clustering coefficients. The so-called "typical" small world is the one with rewiring probability $\mu=0.01$, presenting an average path length almost as low as the random network, while still having a clustering coefficient which is comparable with the one-dimensional regular lattice.

An alternative algorithm proposes to add links instead of rewiring the existing links [22]. This alternative, nonetheless, changes the density of the networks. To ease the comparison between networks, we use the original algorithm, although 

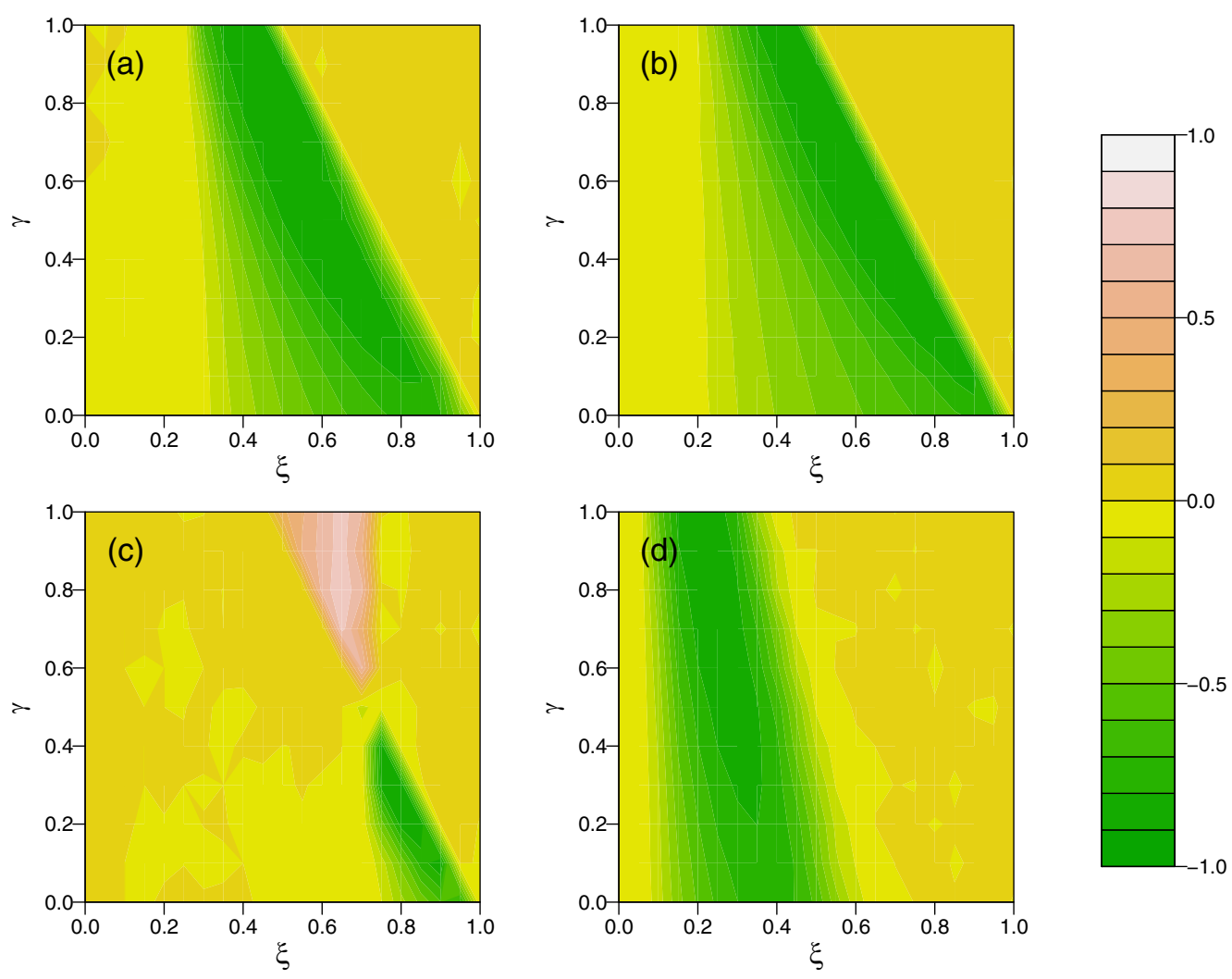

FIG. 3. Difference between the diffusion size in the ring lattice and the random network, $A(\xi, \gamma, \mu=0)-A(\xi, \gamma, \mu=1)$, for (a) a uniform distribution of preferences, (b) an open-minded population, (c) a closed-minded population, and (d) a polarized population. Positive numbers mean the ring lattice gives larger diffusion size than the random network. The results are obtained by averaging the final number of adopters over 50 simulations.

for high rewiring probabilities it can produce disconnected networks.

Figure 2 depicts the interaction of word-of-mouth diffusion with social reinforcement with strong, redundant ties. Once a first adopter enters a neighborhood [Fig. 2(b)], it is likely that other agents in the same neighborhood have a direct tie to the adopting agent. If they adopt, they increase willingness to adopt of the other nodes in the neighborhood due to their added social reinforcement.

\section{SIMULATIONS}

We simulate our extended percolation model of diffusion and compare $A(\xi, \gamma, \mu)$, the final number of adopters of an idea of quality $\xi$ with social influence intensity $\gamma$ in a network with rewiring probability $\mu$. We run the simulations for five small world networks with rewiring probabilities $\mu \in\{0,0.001,0.01,0.1,1\}$, with different degrees of social reinforcement $\gamma \in[0,1]$ and with four different preferences distributions $B(\alpha, \beta)$. All networks have $N=10000$ nodes, each one representing a potential adopter of the idea, with $k=4$ neighbors on average. According to the small world algorithm, in the regular lattice all nodes have degree 4 . These degrees can vary once some of the links are rewired. The MQRs of agents are random draws from a Beta distribution, $q_{0}^{i} \sim B(\alpha, \beta)$. In order to minimize the effect of "lucky draws," the results show the mean percentage of adopters over $R=50$ runs of the same scenario, or combination of network structure and quality of the idea $\xi \in[0,1]$. In all simulations the diffusion process is initialized with ten randomly chosen early adopters, the seeds of the simulation.

We first focus on the results for the two extreme network topologies of a regular ring lattice and a fully random network. Figure 3 compares the final diffusion size between the regular ring lattice and the random network ranging from 1.0 to -1.0 , and for the four different distributions of MQR. Each point of these graphs reports the difference between the final level of adoption in regular and random networks for a certain combination of quality of the idea $\xi$ (horizontal axis) and social influence intensity $\gamma$ (vertical axis), that is to say, $A(\xi, \gamma, \mu=0)-A(\xi, \gamma, \mu=1)$. Positive values (red and white colors) refer to combinations of $\xi$ and $\gamma$ where the ring lattice presents a larger diffusion size than the random network at the end of the simulation.

As a first observation, for most combinations of quality $\xi$ and social reinforcement $\gamma$, random networks reach a diffusion size similar to or higher than the ring lattice. Secondly, we notice a nonmonotonic pattern of diffusion size with respect to the quality of the idea $\xi$ : starting from $\xi=0$, and increasing this quality, the random network becomes relatively more and more efficient than the lattice, but at some point this gap starts to reduce and gets to zero for a quality $\xi=1$. The amplitude of this difference of performance between the two network structures is larger for weaker social influences.

The diffusion results outlined above are common to populations with three MQR distributions, namely, the uniformly 

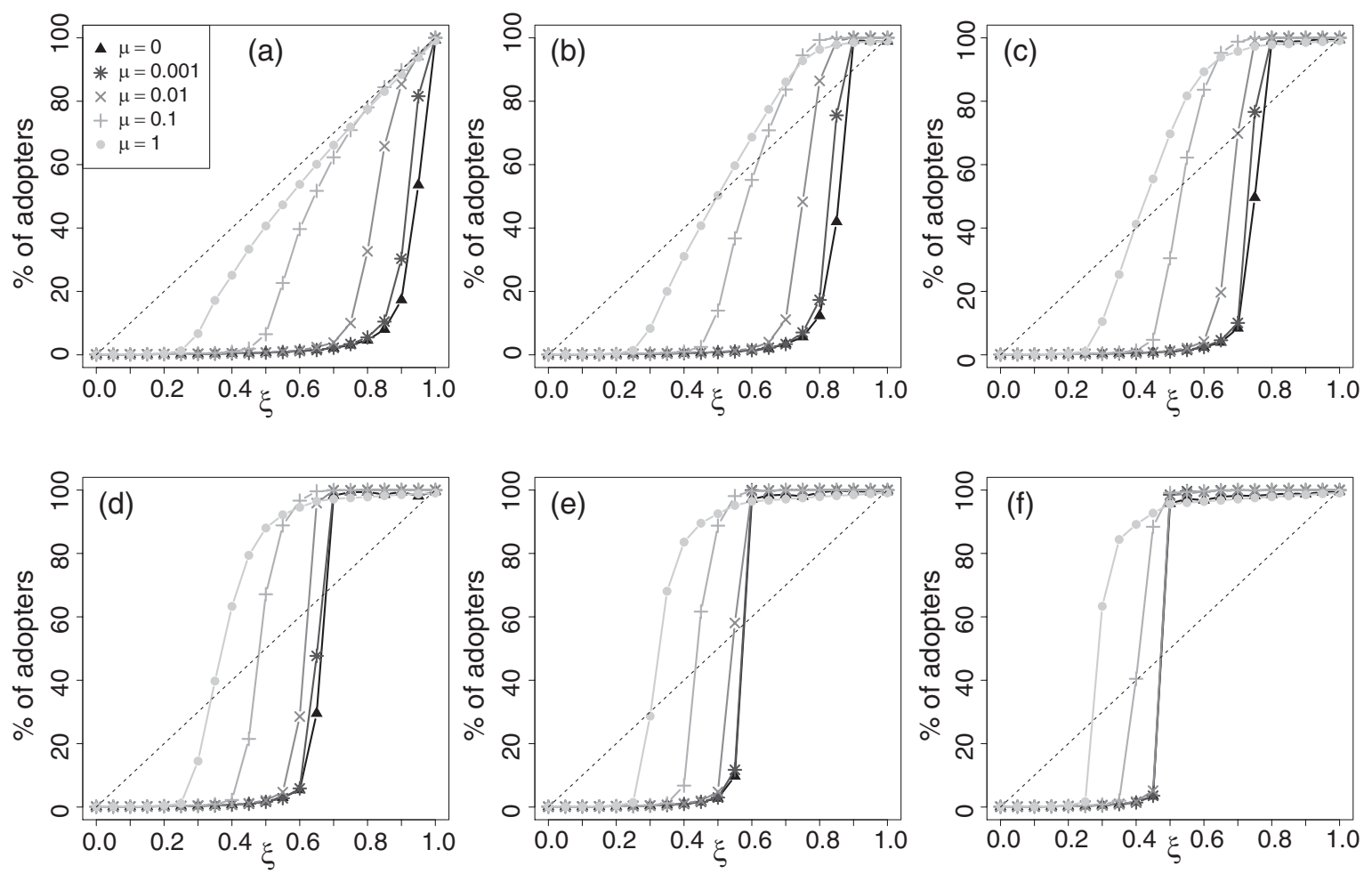

FIG. 4. Adoption rates in a uniform population, $\mathrm{MQR} \sim B(1,1)=U(0,1)$, with social reinforcement intensity (a) $\gamma=0$, (b) $\gamma=0.2$, (c) $\gamma=0.4$, (d) $\gamma=0.6$, (e) $\gamma=0.8$, and (f) $\gamma=1$.

distributed population with $B(1,1)$, the open-minded population with $B(1,4)$, and the polarized population with $B(0.5,0.5)$. A closed-minded population, with a $B(4,1)$ distribution of MQR, presents a different behavior [Fig. 3(c)]. In this case the ring lattice can be more efficient than the random network. This happens for qualities of the idea between 0.5 and 0.8 , and for a social reinforcement strong enough, above $\gamma=0.5$. Again, there is a nonmonotonic pattern: as the quality of the idea increases, the regular lattice becomes more and more efficient in terms of diffusion size, reaching a maximum of relative performance for middle value of the quality of the idea, after which the difference reduces, but remains positive.

We believe these results shed light on the role of different factors in determining diffusion in social networks, and reconcile contradicting results. Previous contributions in the literature suggested that under social reinforcement diffusion in clustered networks such as the ring lattice should outperform diffusion in nonclustered networks such as the random network. Our results show that this only holds true if most of the population would originally be unwilling to adopt (a distribution skewed towards high MQR thresholds). In such a case, diffusion in the random network stops fairly early, since most agents are, after all, unwilling to adopt. In the highly clustered regular lattice, every new adopter in a neighborhood increases the probability of additional neighbors adopting, so that by means of social pressure the adoption process can advance farther than in the nonclustered random network. This interaction of social pressure with clustering in the network is depicted in Fig. 2.

We now study more in depth the effect of clustered network structures, by using the small world model. Figures 4-7 report the diffusion size in five small world networks with different values of the rewiring probability $\mu \in\{0,0.001,0.01,0.1,1\}$ for four different distributions of individual MQR. In this case, we consider six different values of the intensity of social reinforcement $\gamma$.

For a uniform distribution of individual preferences, the less clustered the network is, the better it performs, for all levels of social reinforcement $\gamma$, as shown in Fig. 4. The main driver of diffusion in this case is the "spreading" structure of the network, interpreted as an exponential growth in the connectivity of nodes at successive orders [15,23]: with every additional adopter, the idea can reach a bigger number of additional agents in a random network than in a clustered network. The interaction of clustering with social reinforcement makes the differences between networks decrease as social reinforcement increases. Thus, it is clear that social reinforcement has a stronger effect on the more clustered networks.

Also note that for all networks social reinforcement decreases the threshold at which diffusion in the network starts, as well as the threshold at which full diffusion is reached. This effect is stronger for the more clustered networks, so that the width of the segment of qualities in which we find differences between networks is reduced from around $\xi \in[0.2,1]$ in the case without social reinforcement to around $\xi \in[0.2,0.5]$ in the case with maximum social reinforcement $\gamma=1$.

In an open-minded population, where the distribution of preferences is skewed towards zero (Fig. 5), diffusion results in the different small world networks are similar to the uniform distribution. The ordering of performance for different degrees of clustering is the same. The only difference is that now the percolation thresholds of different networks are closer to 

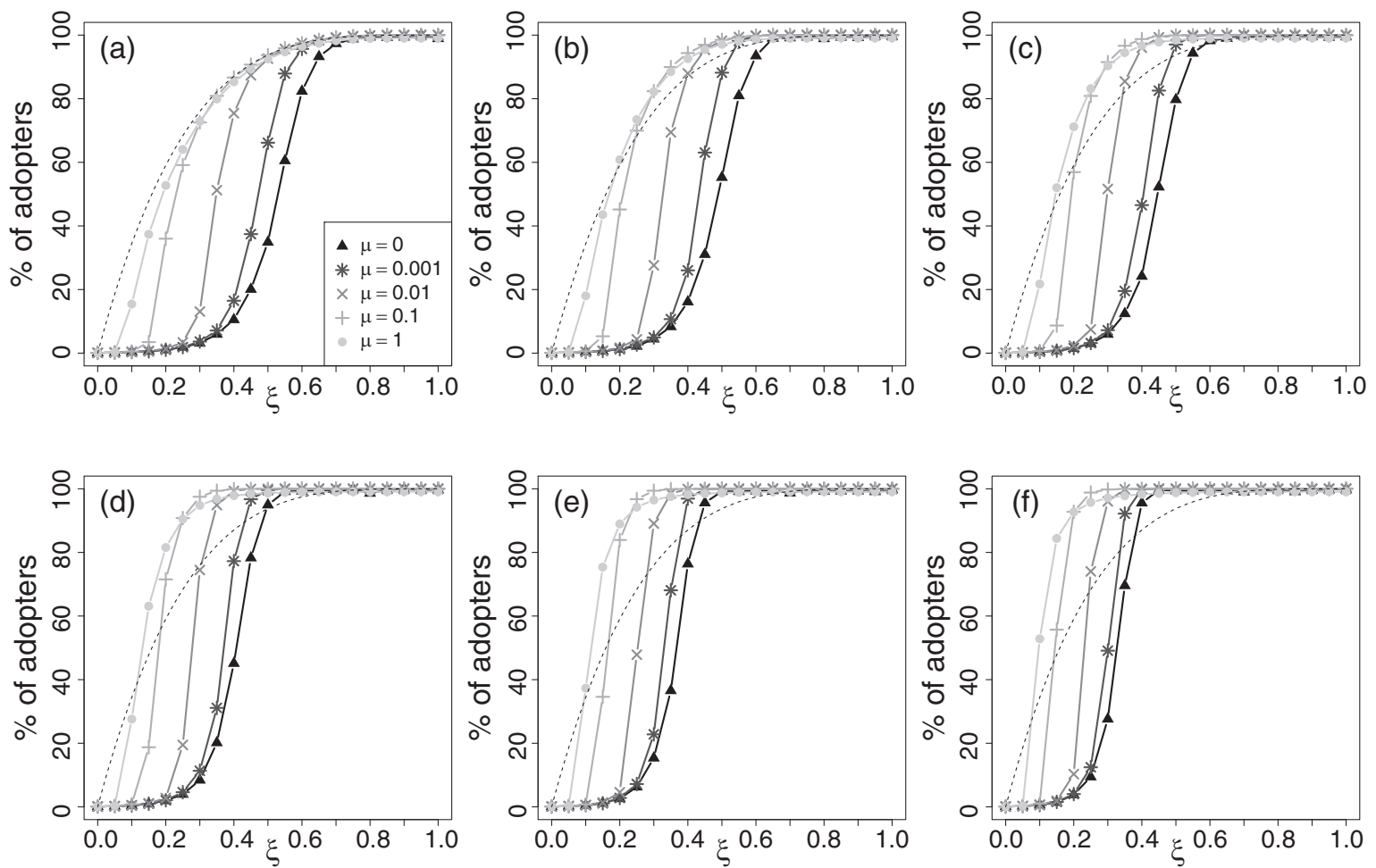

FIG. 5. Adoption rates in an open population, MQR $\sim B(1,4)$, with social reinforcement intensity (a) $\gamma=0$, (b) $\gamma=0.2$, (c) $\gamma=0.4$, (d) $\gamma=0.6$, (e) $\gamma=0.8$, and (f) $\gamma=1$.

each other, and the effect of social reinforcement gets diluted by the fact that there are not so many agents to convince to adopt.
The case of a closed-minded population is again the most interesting one (Fig. 6). Here the MQR thresholds are skewed towards 1 , and follow a $B(4,1)$ distribution. In this case,
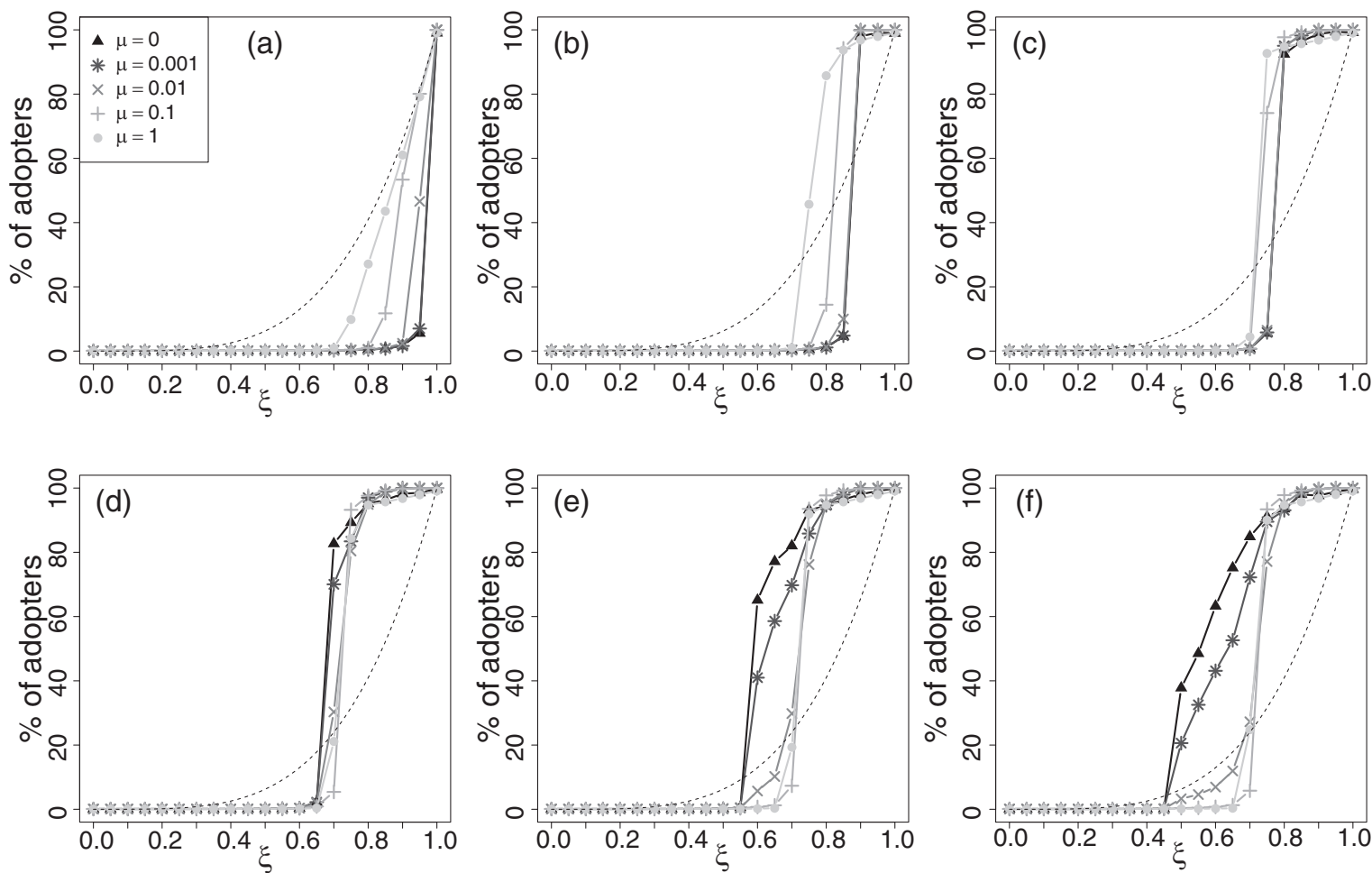

FIG. 6. Adoption rates in a closed-minded population, MQR $\sim B(4,1)$, with social reinforcement intensity (a) $\gamma=0$, (b) $\gamma=0.2$, (c) $\gamma=0.4$, (d) $\gamma=0.6$, (e) $\gamma=0.8$, and (f) $\gamma=1$. 

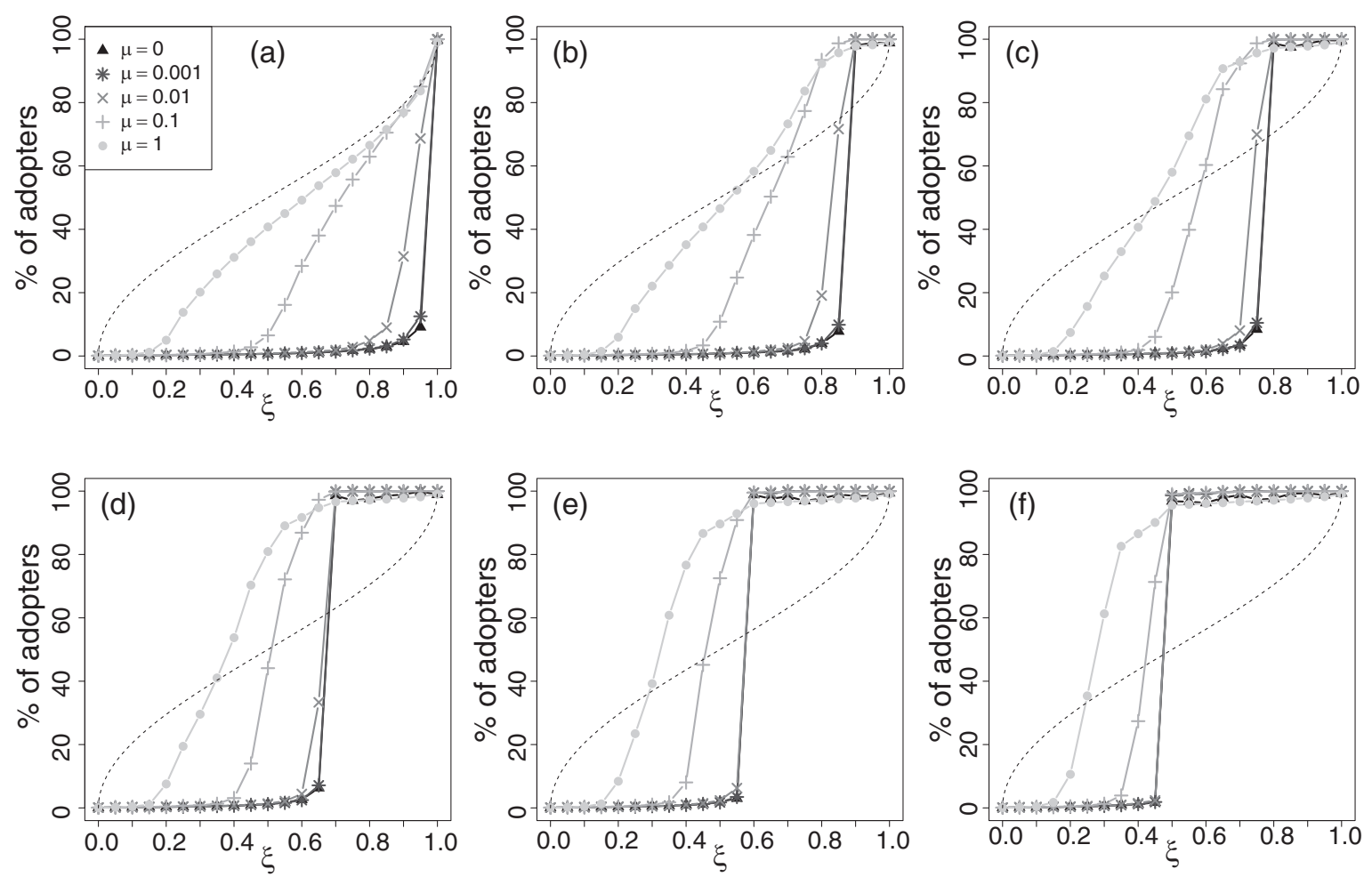

FIG. 7. Adoption rates in a polarized population, $\mathrm{MQR} \sim B(0.5,0.5)$, with social reinforcement intensity (a) $\gamma=0$, (b) $\gamma=0.2$, (c) $\gamma=0.4$, (d) $\gamma=0.6$, (e) $\gamma=0.8$, and (f) $\gamma=1$.

percolation thresholds are very close to each other. But when one increases the intensity of social reinforcement, the clustered network structures outperform random networks. The intuition is as follows. For a population with high probability mass on relatively large values of MQR, most agents do not adopt a new idea unless it is extremely good (with a quality close to 1 ). The random network cannot diffuse the idea very far since diffusion will soon come to dead ends, agents who are not willing to adopt and thus break the chain of diffusion. In the clustered networks, nonetheless, social reinforcement induces a cascade of diffusion and induce neighborhoods of reluctant agents to adopt. Indeed, the effect of social reinforcement is still (as in the previous cases) stronger the more clustered a network is. For a social reinforcement intensity of $\gamma=0.4$, all networks are equivalent in terms of diffusion size. From there, the more clustered a network is, the higher its diffusion size.

The last question to address is whether the result obtained for a closed-minded population is driven by the high portion of willing to adopt agents or by the low portion of unwilling to adopt agents. The last set of simulations (Fig. 7) addresses this question, by looking at a polarized distribution such as the $B(0.5,0.5)$. For such a population most of the probability mass of MQR is either at low values close to zero or high values close to one. While the effect of social reinforcement is stronger for the more clustered networks, the random network still outperforms all the others. This means that having a sizable mass of agents with high values of MQR does not "do the trick." Put differently, the relevant quantity for complex propagation processes to diffuse better in clustered networks is more the absence of a pool of willing to adopt agents, rather than the existence of a pool of unwilling to adopt agents.
Our results send the following message: diffusion in a social network depends on the combined effect of clustering of social contacts, the possible reinforcement effect of social influence, and the distribution of individual preferences. Clustered networks, such as small world networks, are relatively efficient when social influence is strong enough, but only for the particular distribution of preferences where there is a larger mass of agents with a high individual threshold for adoption.

\section{CONCLUSION}

Diffusion often occurs through social contact. The topology of the diffusion process is thus that of the social network in which it takes place. A direct question, then, is how the structure of the network affects the outcome of the diffusion process. That is to say, which network topologies facilitate diffusion, and which delay it or reduce its final breadth? The debate has recently moved on from how the network structure affects the diffusion of "whatever is being diffused" to how different disseminules diffuse in different network structures. It has been found [8] that weak ties and strong ties have a different role simple propagation processes where one contact with the disseminule is sufficient to trigger adoption, as compared to complex propagation processes, where additional contacts are not redundant but reinforce the probability of contagion.

This paper goes one step further and analyzes how these factors interact with individual preferences of the agents in the population in which the disseminule is being diffused. Weak ties act as bridges for the diffusion of simple propagation, and clustering ties reinforce the diffusion of disseminules for which additional contacts increase the likelihood of contagion. 
Nonetheless, as we show, this does not mean that random networks perform best in simple propagations and clustered networks perform best in complex propagations, as argued before [8]. Only if the population is substantially biased against adoption of the disseminule will clustered networks outperform random networks in complex propagation processes. This result underscores the need-both in theoretical models and empirical research-to take into account the heterogeneity of preferences of agents involved in diffusion processes.
[1] M. E. J. Newman, SIAM Rev. 45, 167 (2003).

[2] M. Granovetter, Am. J. Sociol. 78, 1360 (1973).

[3] C. Moore and M. E. J. Newman, Phys. Rev. E 61, 5678 (2000).

[4] A.-X. Cui, Z. Yang, and T. Zhou, Physica A 445, 335 (2016).

[5] L. Lü and T. Zhou, Europhys. Lett. 89, 18001 (2010).

[6] D. Centola, V. M. Eguiluz, and M. W. Macy, Physica A 374, 449 (2007).

[7] M. Granovetter, Am. J. Sociol. 83, 1420 (1978).

[8] D. Centola and M. Macy, Am. J. Sociol. 113, 702 (2007).

[9] D. Centola, Science 329, 1194 (2010).

[10] M. Zheng, L. Lü, and M. Zhao, Phys. Rev. E 88, 012818 (2013).

[11] P. L. Krapivsky, S. Redner, and D. Volovik, J. Stat. Mech.: Theor. Exp. (2011) P12003.

[12] S. Solomon, G. Weisbuch, L. de Arcangelis, N. Jan, and D. Stauffer, Physica A 277, 239 (2000).

[13] M. Hohnisch, S. Pittnauer, and D. Stauffer, Industrial and Corporate Change 17, 1001 (2008).
[14] S. Cantono and G. Silverberg, Technological Forecasting and Social Change 76, 487 (2009).

[15] P. Zeppini and K. Frenken, Networks, Percolation, and Demand, Bath Economic Research Papers Vol. 38/15 (Department of Economics, University of Bath, UK, 2015).

[16] L. Lü, D.-B. Chen, and T. Zhou, New J. Phys. 13, 123005 (2011).

[17] T. S. Kuhn, The Structure of Scientific Revolutions, 4th ed. (Chicago University, Chicago, 2012).

[18] E. M. Rogers, Diffusion of Innovations, 5th ed. (Free Press, New York, 2003).

[19] R. Kemp, J. Schot, and R. Hoogma, Technology Analysis and Strategic Management 10, 175 (1998).

[20] D. J. Watts and S. H. Strogatz, Nature (London) 393, 440 (1998).

[21] P. Erdös and A. Rényi, Publicationes Mathematicae 6, 290 (1959).

[22] M. E. J Newman and D. J. Watts, Phys. Rev. E 60, 7332 (1999).

[23] R. Albert and A. L. Barabási, Rev. Mod. Phys. 74, 47 (2002). 